\title{
Antidepressant treatment worsens metabolic control in type 2 diabetes mellitus
}

Fernández-García, José Carlos (1), Cortés-Salazar, Carmen María (2)

1. Endocrinology Department. Virgen de la Victoria University Hospital. Málaga (Spain).

2. Badolatosa Primary Care Centre. Seville (Spain).

\section{Objectives}

To evaluate the use of antidepressant drugs in patients with type 2 diabetes mellitus (T2DM) and to analyze if these drugs are associated with a more deteriorated metabolic control.

\section{Methods}

- Cross-sectional study

- Inclusion criteria: T2DM patient followed in a primary care setting.

- Data about age, sex, T2DM evolution, body mass index (BMI), HbA1c and associated antidepressant treatment was collected.

Statistical analysis was performed with SPSS v 15.0 for Windows.

\section{Basal characteristics ( $\mathbf{n - 7 9 )}$}

\begin{tabular}{|l|c|}
\hline Age (years) & $70.1 \pm 11.8$ \\
Male (\%) & 63.3 \\
BMl (kg/m²) & $30.8 \pm 11.8$ \\
T2DM duration (years) & $5.9 \pm 5$ \\
Hba1c (\%) & $6.8 \pm 0.9$ \\
Triglycerides (mg/dl) & $148.3 \pm 81.1$ \\
Cholesterol (mg/dl) & $180.5 \pm 40.6$ \\
LDL-cholesterol (mg/dl) & $101.3 \pm 34.7$ \\
HDL-cholesterol (mg/dl) & $49.8 \pm 13.5$ \\
\hline
\end{tabular}

\section{Associated antidepressant treatment (\%)}

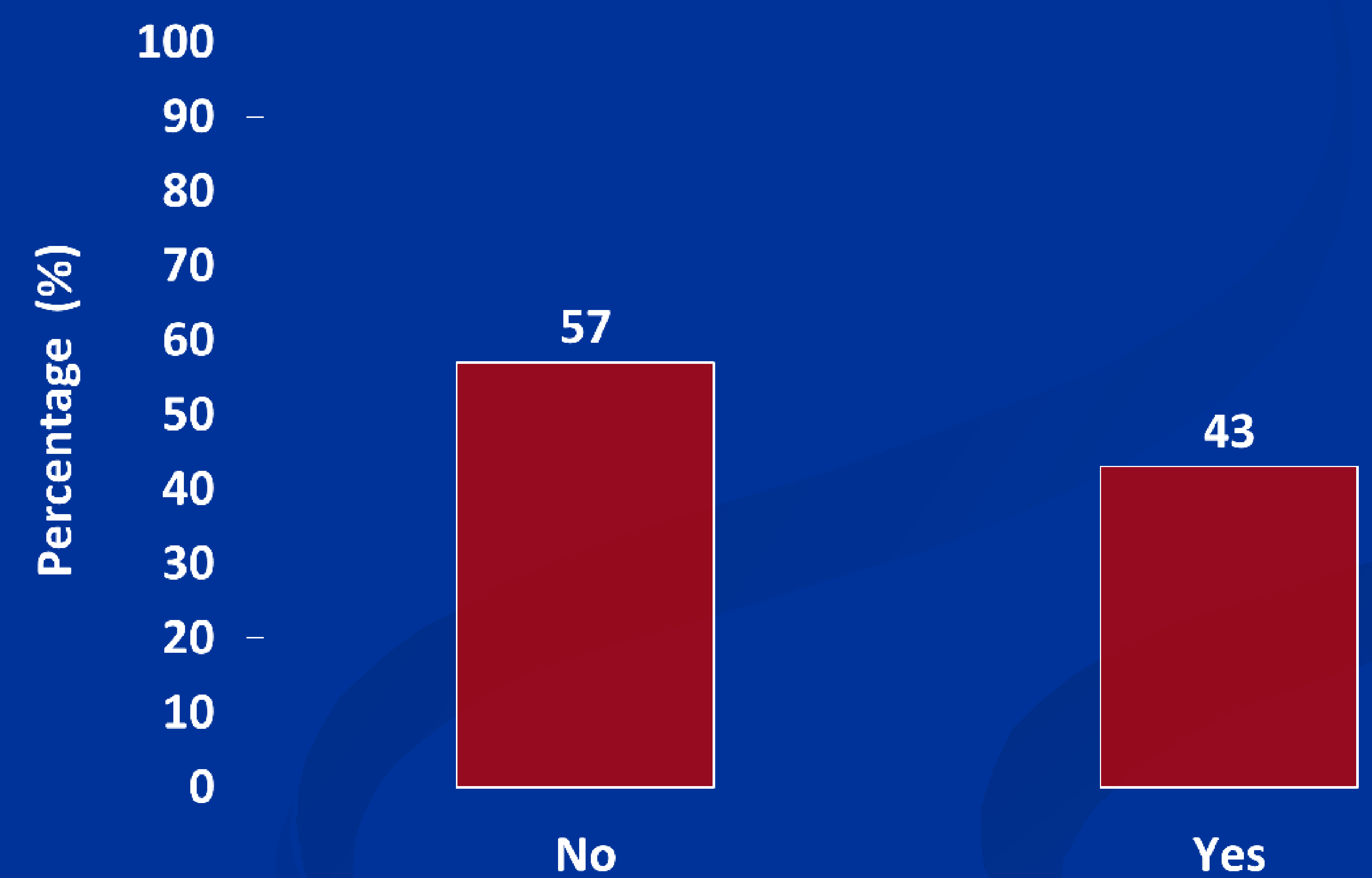

\section{Clinical characteristics by associated antidepressant treatment (\%)}

\begin{tabular}{|l|c|c|c|}
\hline & $\begin{array}{c}\text { Antidepressant } \\
\text { treatment } \\
\mathrm{n}-45\end{array}$ & $\begin{array}{c}\text { Antidepressant } \\
\text { treatment }+\end{array}$ & $\mathrm{p}$ \\
\hline Age (years) & $69.4 \pm 13.4$ & $\mathrm{n}-34$ & \\
Male/Female (\%) & $70 / 35$ & $30.8 \pm 9.4$ & $\mathrm{~ns}$ \\
$\mathrm{BMI}\left(\mathrm{kg} / \mathrm{m}^{2}\right.$ ) & $29.7 \pm 5.2$ & $32.5 \pm 6$ & 0.018 \\
T2DM duration (years) & $5.9 \pm 4.9$ & $5.9 \pm 5.1$ & 0.046 \\
Hba1c (\%) & $6.6 \pm 0.8$ & $7.1 \pm 0.9$ & 0.037 \\
Triglycerides (mg/dl) & $135.7 \pm 59.9$ & $165.7 \pm 101.9$ & 0.11 \\
Cholesterol (mg/dl) & $177 \pm 40.5$ & $185 \pm 40.8$ & $\mathrm{~ns}$ \\
LDL-cholesterol $(\mathrm{mg} / \mathrm{dl})$ & $100.4 \pm 35$ & $102.6 \pm 34.7$ & $\mathrm{~ns}$ \\
HDL-cholesterol $(\mathrm{mg} / \mathrm{dl})$ & $49.9 \pm 13.5$ & $49.6 \pm 13.7$ & $\mathrm{~ns}$ \\
\hline
\end{tabular}

\section{Conclusions}

A A high rate of prescription of antidepressant drugs is frequently found in individuals with T2DM, especially in women.

$\Rightarrow$ T2DM patients under antidepressant treatment have a more deteriorated metabolic control. 